\title{
OBESITY INDUCED BY A SUCROSE-RICH DIET PROMOTES DEFICITS IN BONE MINERALIZATION AND MICROARCHITECTURE
}

LOUZADA, Mário Jefferson Q. ${ }^{1}$; NUNES, M.T. ${ }^{2}$; CARVALHO, A.A.F. ${ }^{3}$; COLLI, V.C. ${ }^{3}$; DORNELLES, R.C.M. ${ }^{3}$; NAKAMUNE, A.C.M.S. ${ }^{3}$; FLORINDO, P.L. ${ }^{1}$ BIFFE, B.G. ${ }^{3}$

${ }^{1}$ Department of Support, Animal Production and Health, Araçatuba School of Veterinary Medicine, UNESP - Univ Estadual Paulista; ${ }^{2}$ Department of Physiology and Biophysics , Institute of Biomedical Sciences, USP - University of São Paulo; ${ }^{3}$ Department of Basic Sciences, Araçatuba Dental School, UNESP - Univ Estadual Paulista

Brazil

Introduction

Obesity and osteoporosis, chronic, non transmissible diseases in public health, share increasingly relevant characteristics and prevalence. Although studies have attributed the positive effect of obesity on bone tissue parallel to this, studies indicating the contrary have appeared.

The aim of this study was to evaluate the effects of obesity induced by sucrose ingestion, on the body mass, glycemia and biometry, as well as to determine the plasma concentration of leptin and insulin, and evaluate biophysical parameters of the bone tissue of Wistar rats.

Methods

\section{Animals}

The experiment is in accordance with the ethical principles of Animal Experimentation and was approved by the Ethics Commission on Animal Experimentation (CEEA) - Protocol No. 2008-002809.

Twenty male Wistar rats, 3 months old, of the Rattus novegicus albinus breed, supplied by the Vivarium of the School of Dentistry, UNESP - São Paulo State University, Araçatuba, Brazil, were randomly selected. The animals were kept in a acclimatized environment $\left(21 \pm 2^{\circ} \mathrm{C}\right)$ and light/dark cycle (12/12 hours/day) and free access to Purina- Labina ${ }^{\circledR}$ rations and water. Randomly, the animals were divided into two experimental groups 10 rats in each: $\mathbf{C}$ - Control Group; $\mathbf{S}$ - Sucrose group that received sucrose solution prepared daily at a concentration of $30 \%(\mathrm{p} / \mathrm{v})$, for eight $(8)$ weeks.

\section{DXA}

Densitometric evaluation was performed at the beginning of the experiment, at the time the two groups were separated, and again after the animals were sacrificed. For the initial densitometric evaluation, the animals were anesthetized by intramuscular administration of ketamine $(0.07 \mathrm{ml} / 100 \mathrm{~g}$ bw/ip - Fort Dodge Saúde Animal Ltda, Brazil) and xilazina $(0.03 \mathrm{ml} / 100 \mathrm{~g}$ bw/ip - Dopaser Laboratories Calier S.A., Barcelona, Spain). Afterwards the animals were placed in a head-to-tail position with the tibia aligned with the laser light beam of the appliance to begin image capture. The tibias were submitted to densitometric analysis by dual energy x-ray absorptiometry (DXA) by means of model DPX-ALPHA LUNAR densitometer, with special software for small animals, with high resolution, with the tibia bone as a whole being scanned for subsequent analysis of results.

\section{$\mu \mathrm{CT}$}

Bone microtomography was performed by means of a computerized microtomograph Skyscan 1172 (Skyscan, Aartselaar, Belgium) used for non destructive tridimensional evaluation of bone architecture. The spongy bone region of interest was manually interpolated, and 100 slices below the growth plate were analyzed at a resolution of $12 \times 12 \times 12 \mu \mathrm{m}(16)$. The tibia was placed in a head-to-tail position for analysis. The parameters analyzed were as follows: BV/TV (\%) - trabecular bone volume as a percentage of tissue volume; Tb.Th $(\mu \mathrm{m})$ - trabecular thickness; Tb. N (1/mm) number of trabeculae per millimeter of tissue, and $\mathrm{Tb} . \mathrm{Sp}(\mu \mathrm{m})-$ trabecular separation.

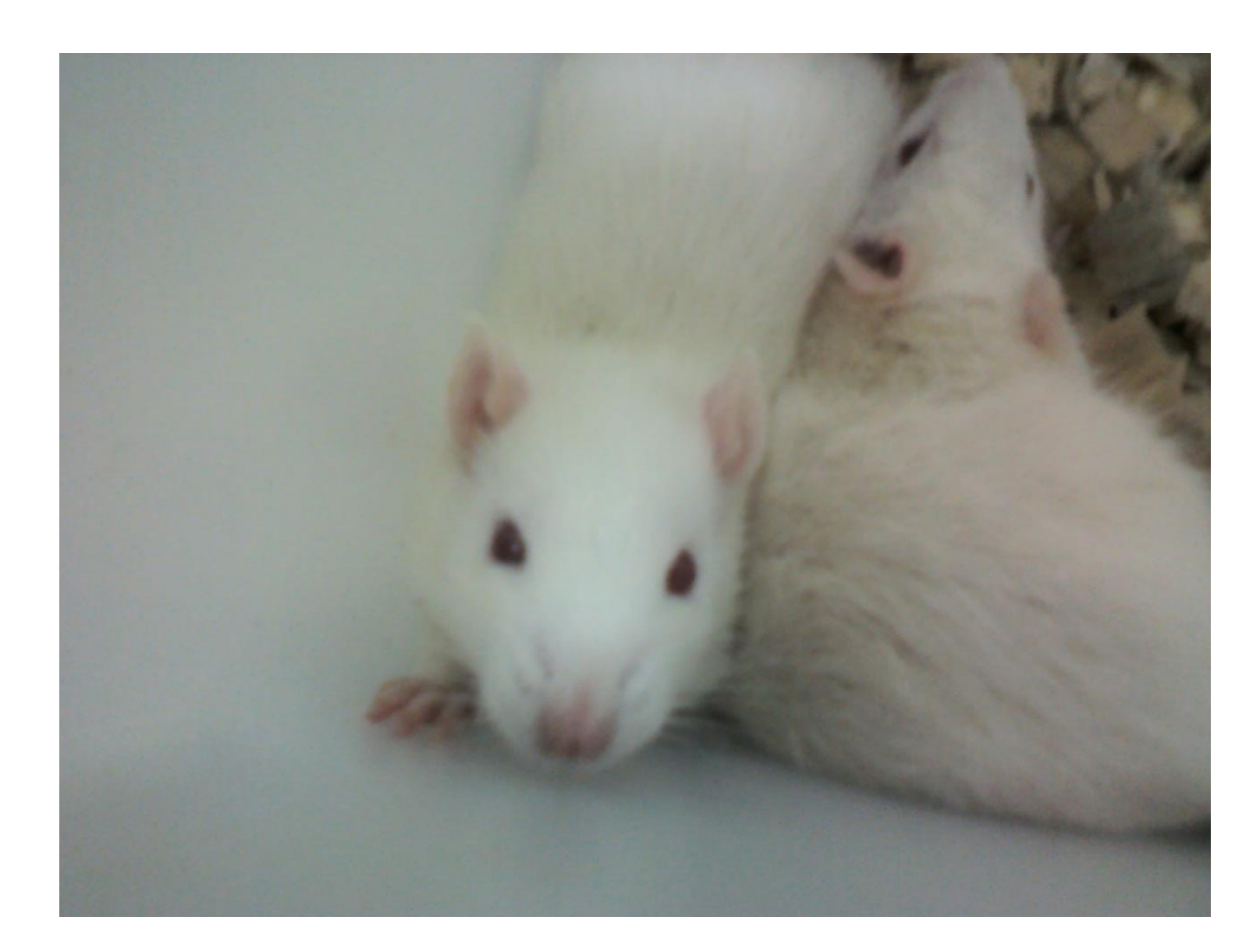

OBESITY X BONE

Results


Control

Sucrose

Conclusion

In view of the results obtained, it was concluded that obesity induced by means of a sucroserich diet promoted a change in the dietary behavior of the rats, with a consequent reduction in bone mineralization, in addition to negatively influencing their bone microarchitecture. 